\title{
THE STIR SEQUENCE MRI IN THE ASSESSMENT OF EXTRAOCULAR MUSCLES IN THYROID EYE DISEASE
}

\author{
H. B. $\mathrm{HOH}^{1}$, R. D. LAITT ${ }^{2}$, C. WAKELEY ${ }^{2}$, J. KABALA ${ }^{2}$, P. GODDARD ${ }^{2}$, M. J. POTTS ${ }^{1}$ \\ and R. A. HARRAD \\ Bristol
}

\begin{abstract}
SUMMARY
Nineteen patients with thyroid eye disease were examined with magnetic resonance imaging using the Short Tau Inversion Recovery (STIR) sequence and compared with normal controls. The Signal Intensity Ratio (SIR) of each of the four recti was obtained by comparison with the signal intensity of the adjacent temporalis muscle. The SIR was compared with disease activity assessed using Werner's grading system, a clinical inflammatory score, and the range of extraocular movements. Results show that a high SIR is associated with a high index of disease activity. This technique is likely to prove useful in assessing disease activity and planning the management of thyroid eye disease, particularly with respect to immunosuppression.
\end{abstract}

Thyroid eye disease occurs in $2-7 \%$ of patients with Graves' disease.' It is an autoimmune disorder characterised by lymphocytic infiltration in the early phase..$^{2-4}$ The clinical presentation ranges from mild disease with puffiness around the eyes, lid lag and lid retraction to severe disease with gross exophthalmos with conjunctival injection, limited extraocular movements and visual loss due to optic nerve compression as the disease progresses. ${ }^{5}$ There appear to be two stages in the disease process which differ both clinically and immunologically: in the active ('wet') phase the eye is chemotic and hyperaemic and becomes progressively more uncomfortable and sore, whilst in the quiescent ('dry' or burnt-out) phase the eye is white with stable non-progressive signs. ${ }^{6}$ Pathologically the initial active 'wet' phase is associated with an inflammatory infiltrate dominated by lymphocytic infiltration of extraocular muscles associated with the accumulation of glycoaminoglycans and tissue oedema. Although the

From: 'Bristol Eye Hospital, Bristol; ${ }^{2}$ Department of Diagnostic Radiology, Bristol Royal Infirmary, Bristol, UK.

Correspondence to: H. B. Hoh, Bristol Eye Hospital, Lower Maudlin Street, Bristol BS1 2LX, UK. majority of these cases resolve with the regression of the inflammation, some progress to develop sight-threatening compressive optic neuropathy and the patient be left with intractable diplopia.

Low-dose orbital radiotherapy has been used with considerable success in alleviating the inflammatory process of thyroid eye disease. ${ }^{8.9}$ More recent studies have shown that better results are achieved if radiotherapy is used in early disease when the soft tissue signs are maximal, reflecting the radiosensitivity of the autoimmune inflammatory infiltrate at the early active stage. ${ }^{10-12}$

Although conventional magnetic resonance imaging (MRI) is the ideal medium for discerning soft tissue pathology according to its hydrogen content, it is unable to discriminate fat from water-containing tissues, which appear bright on both T1- and T2-weighted MRI images. The background stroma of the orbit is composed mainly of fat and has a characteristic bright signal in conventional spinecho (SE) MRI. Short Tau (inversion time) Inversion Recovery (STIR), by summation of T1 and T2 and by choosing the appropriate inversion time (TI), can selectively suppress signal from a tissue such as fat whilst highlighting water-containing tissues. An acute inflammatory process is associated with oedema within the tissue and would be expected to show a bright signal in the STIR sequence in the MRI. The aim of this study was to evaluate the use of the STIR sequence in MRI of the orbit as an indicator of disease activity in thyroid eye disease.

\section{METHODOLOGY}

Orbital changes in 19 patients with a history of hyperthyroidism presenting with varying stages of dysthyroid eye disease were examined with MRI. The mean age of these patients was 48 years with a range between 26 and 73 years. Thirteen patients were female while 6 were male. The duration of thyroid disease ranged from 4 to 144 months with a mean of 45 months. The mean duration of 
thyroid eye disease, however, was 9 months (range 2-36 months). Five controls with a mean age of 41 years were selected at random from patients undergoing MRI imaging of the brain and who had no ocular disease.

Patients were questioned regarding symptoms of discomfort, foreign body sensation, lacrimation, photophobia and dryness. These were graded as mild, moderate or severe and scored as 1,2 or 3 respectively. Assessment of the patients' clinical states included pupillary reactions, measurement of proptosis in millimeters using a Hertel exophthalmometer, estimation of the range of extraocular movement, measurement of the field of uniocular movement and binocular single vision. Visual function was assessed from the visual acuity, Ishihara pseudoisochromatic chart and the presence of an afferent pupillary defect. These ocular changes were then graded in accordance with the American Thyroid Association classification as described and later modified by Werner. ${ }^{13}$ In addition, the amount of active inflammation was assessed clinically by the degree of hyperaemia and chemosis, and was scored as 1 (mild), 2 (moderate) or 3 (severe). This mirrors closely those parameters suggested by Mourits et $a l .{ }^{14}$ whose grading scale tries to emphasise the differentiation between the 'wet' and 'dry' phases.

Imaging was performed on a $1.0 \mathrm{~T}$ scanner (Siemens Magnetom Impact) with a head coil that covered both eyes. All patients underwent both spin-echo (SE) and STIR imaging with T1-weighted (TR/TE: 500/15 ms) and STIR (TR/TE/TI: 3000/20/150 ms) images obtained in the coronal plane and multiecho (TR/TE: 2200/20 and $80 \mathrm{~ms}$ ) images in the axial plane. STIR images were quantified by measuring the signal intensity of individual extraocular muscles by pixel analysis of their brightness. These measurements were then compared with the signal intensity of the ipsilateral temporalis muscle and expressed as a ratio. This signal intensity ratio (SIR) was used in preference to an absolute figure due to slight variation of sensitivity of the machine when used on subsequent occasions. The temporalis muscle was selected because of its structural similarity and close anatomical proximity to the extraocular muscles in order to maximise error and variation secondary to positioning within the magnetic field.

Regression analysis was performed to compare the mean SIR of the four extraocular muscles with Werner's grade of the thyroid eye disease and the clinical activity score. In addition, the mean SIR of the horizontal and vertical recti were compared with the range of horizontal and vertical extraocular muscle movements respectively.

\section{RESULTS}

The control patients had an SIR of individual extraocular muscle which ranged from 1.16 to 1.49 with a mean of $1.38(\mathrm{SD}=0.1)$. The mean SIR was 1.39 in the right and 1.36 in the left (Table I).

Of the 19 patients with dysthyroid eye disease, 1 had stage 1 disease, 4 had stage 2 disease, 8 had stage 4 disease, 1 had stage 5 disease while 5 had stage 6 disease. Their SIR ranged from 1.2 to 2.18 with a mean of 1.59 in
Table I. Mean extraocular muscle signal intensity ratio (SIR)

\begin{tabular}{lcc}
\hline & Right & Left \\
\hline $\begin{array}{l}\text { Controls } \\
\text { (range 1.16-1.49) }\end{array}$ & 1.39 & 1.36 \\
$\begin{array}{l}\text { Graves' disease } \\
\text { (range 1.2-2.18) }\end{array}$ & 1.59 & 1.64 \\
\hline
\end{tabular}

the right and 1.64 in the left (Table I). Areas of increased SIR (which we took as more than 1.49 or 1 SD from the mean SIR of the controls) were found in the extraocular muscles of 13 of the 19 patients $(62 \%)$ and were bilateral in 8 cases. All four rectus muscles were affected; inferior rectus involvement was the most frequent $(8$ cases or $42 \%)$, the medial rectus being involved in 6 cases (31\%) and the superior rectus in just over a quarter of the cases. The lateral rectus appeared relatively spared. The STIR sequence in the MRI of a patient with dysthyroid eye disease affecting predominantly the left inferior rectus is shown in Fig. 1.

The mean SIR of the orbit showed a positive correlation (correlation coefficient of +0.71 and $p<0.001$ ) with the disease activity staged according to the Werner classification (Fig. 2). The signal intensity of the patients with

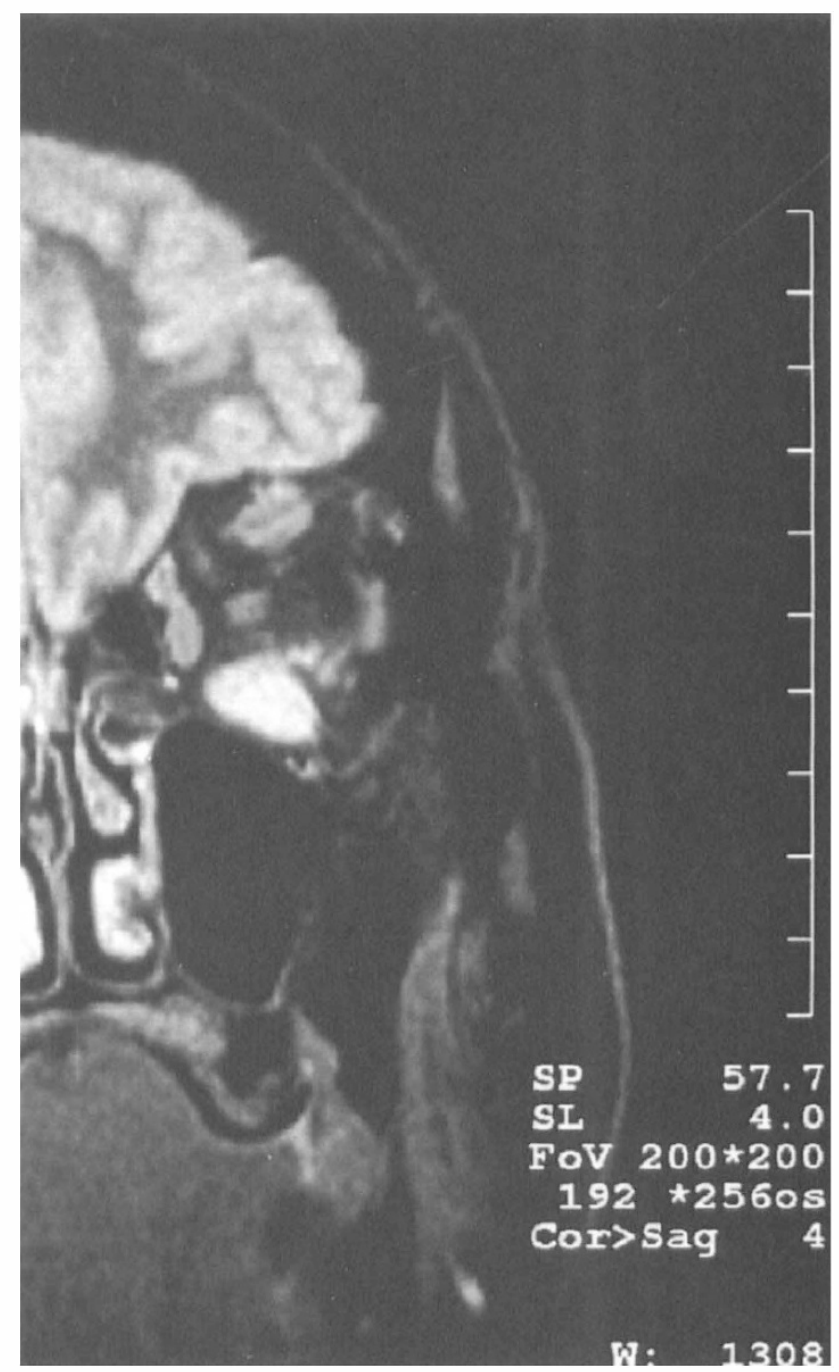

Fig. 1. The STIR sequence in the MRI of a patient with dysthyroid eye disease affecting predominantly the left inferior rectus. 


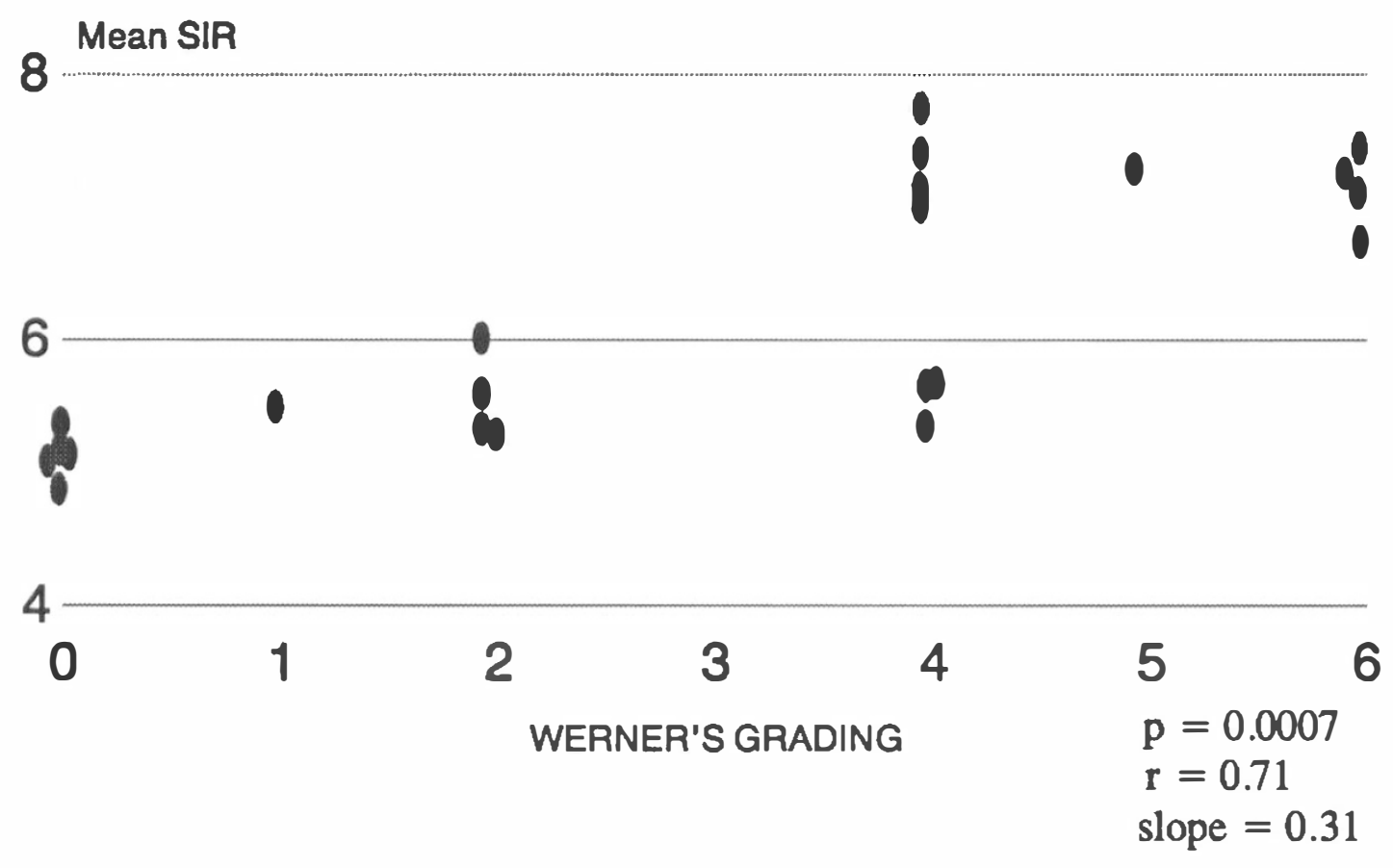

Fig. 2. Correlation between mean signal intensity ratio $(S I R)$ and Werner's grading.

\section{Mean SIR}
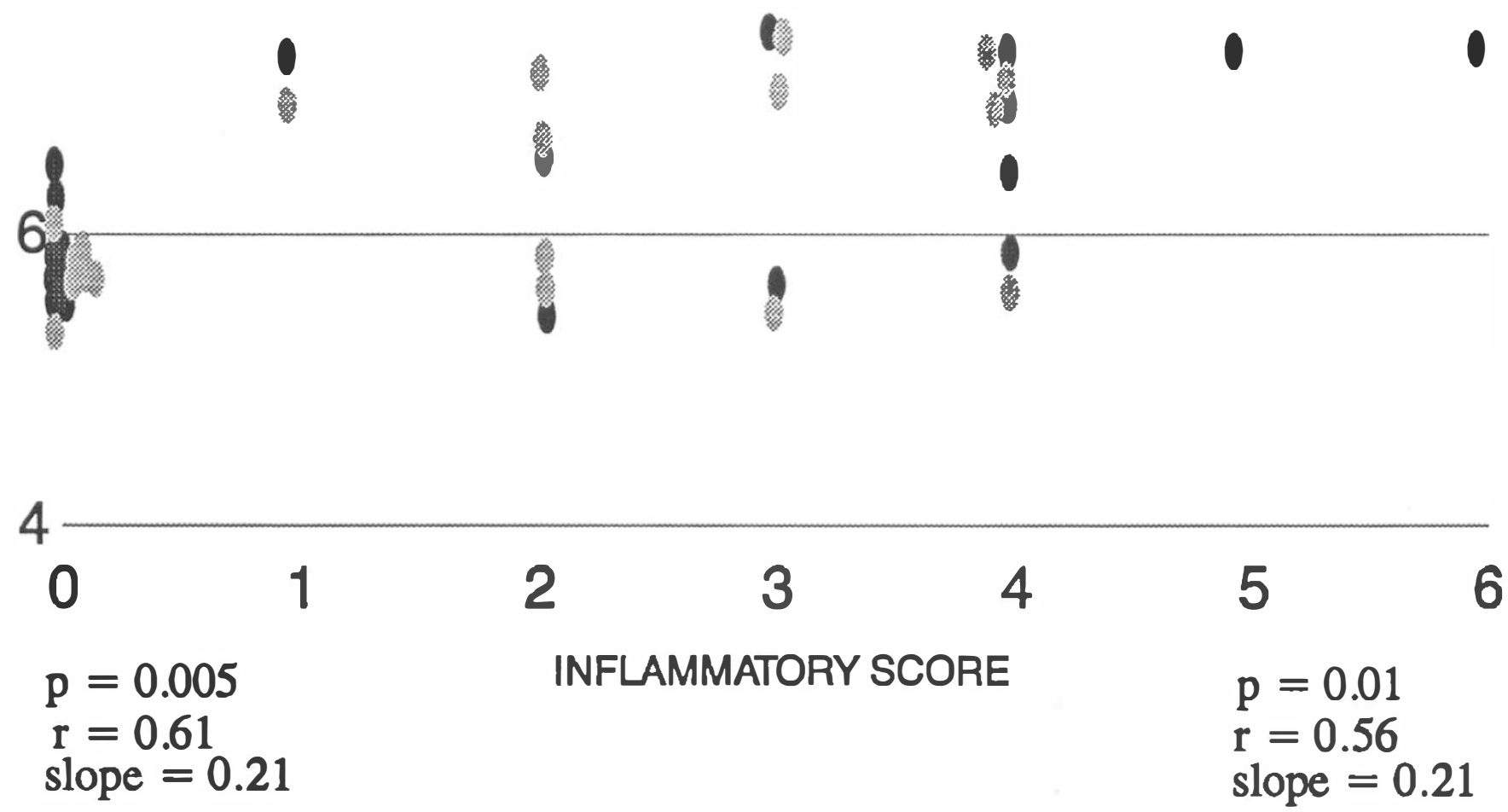

Fig. 3. Correlation between mean SIR and inflammatory score. Black ovals, right eye; grey ovals, left eye. 


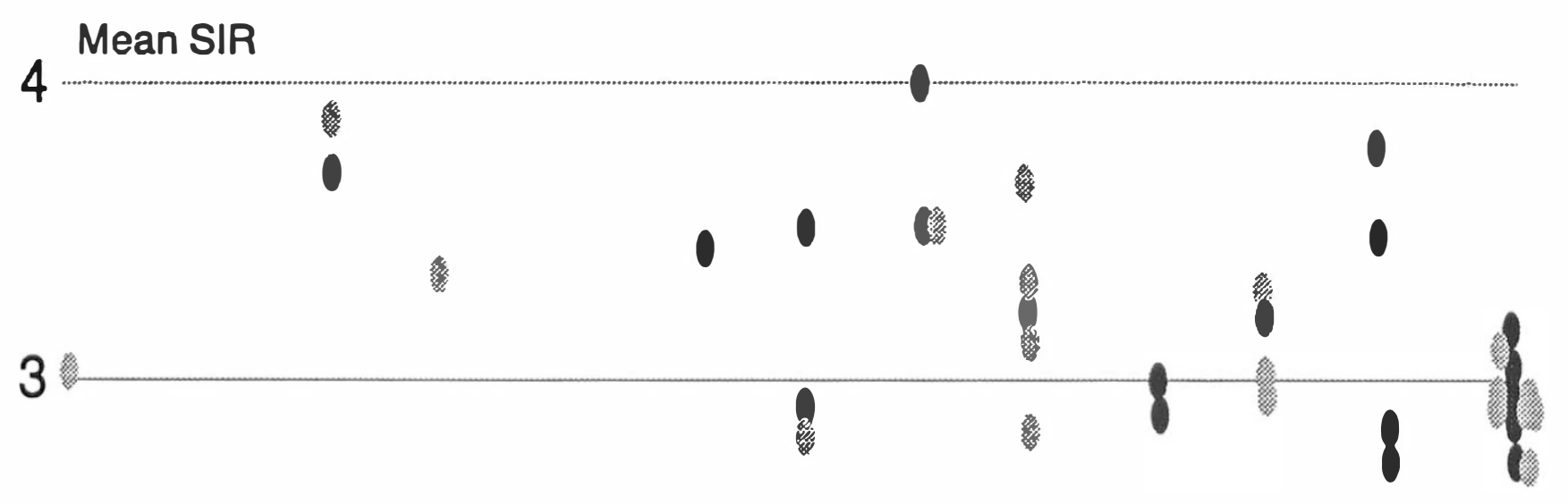

2

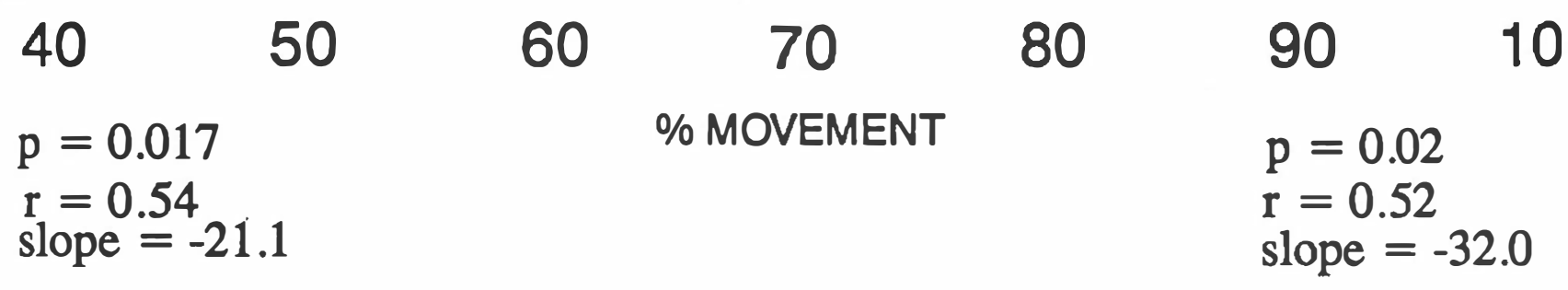

Fig. 4. Correlation between mean SIR and horizontal movement. Black ovals, right eye; grey ovals, left eye.

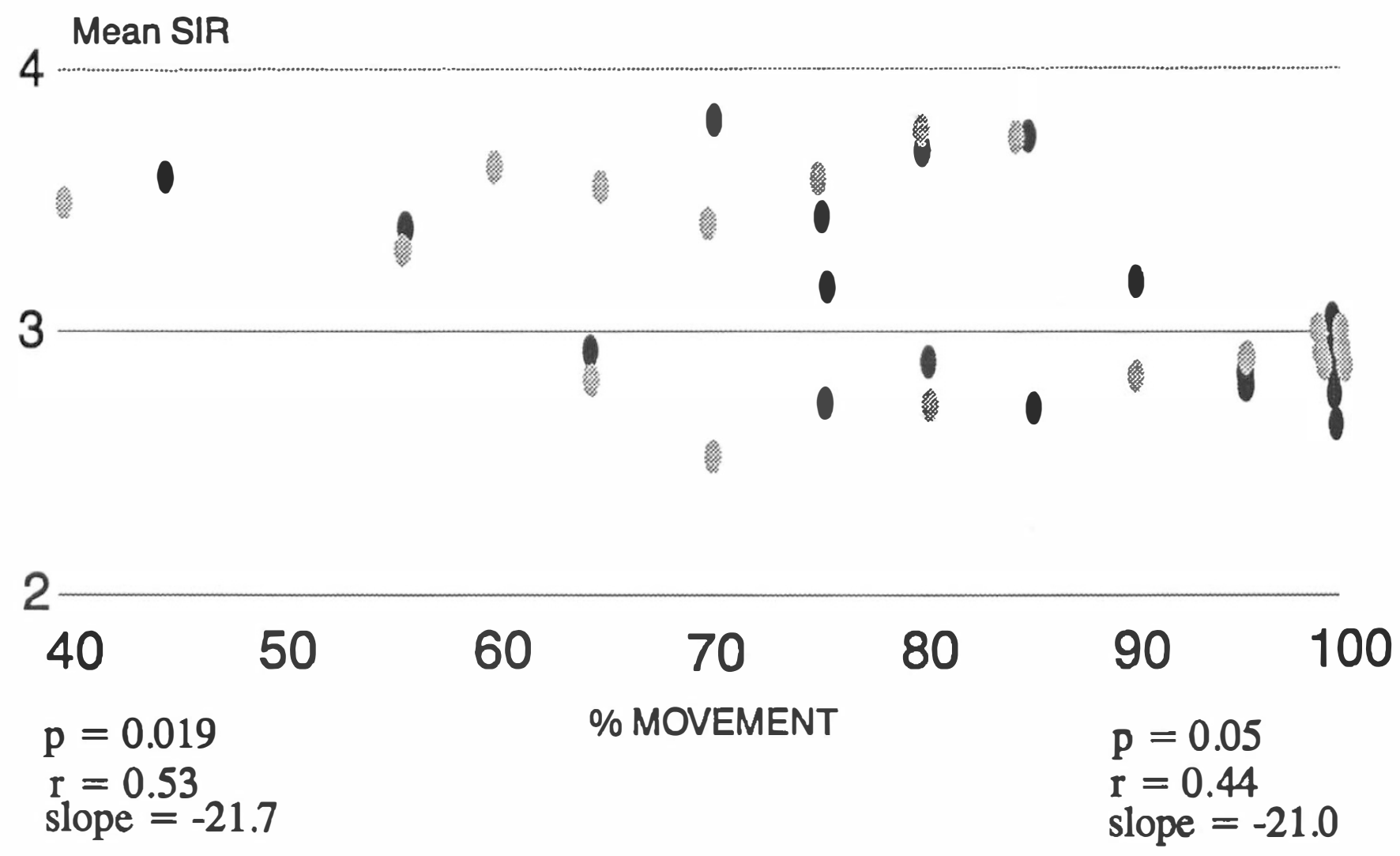

Fig. 5. Correlation between mean SIR and vertical movement. Black ovals, right eye; grey ovals, left eye. 
stage 1-2 disease was comparable to the control (stage 0 ), while stage 5 and 6 disease states exhibited a marked increase in SIR. The extraocular muscles in stage 4 disease (i.e. patients with extraocular muscle involvement) produced a mixed picture, with some patients having near normal and others having an increased signal intensity.

Fig. 3 shows a positive correlation between mean SIR of each orbit and inflammatory score, with a correlation coefficient of +0.61 and +0.56 for the right and left orbit respectively $(p<0.01)$.

The range of extraocular movement was evaluated as a percentage of full duction and was compromised in varying degrees in dysthyroid patients. Values ranged from 5\% to $100 \%$ of normal limits. When the range of movements were compared with the SIR, there was a negative correlation between the range of horizontal and vertical duction, and the mean SIR of the horizontal (i.e. medial and lateral rectus) and mean vertical (i.e. superior and inferior rectus) groups of extraocular muscles respectively (Figs. 4, 5). This suggests that there is an acute inflammatory process within the extraocular muscles leading to restriction of movement which manifests as a bright signal on the STIR sequence, and which correlates with the clinical activity score.

No relation was established between the duration of either the underlying thyroid disorder or thyroid eye disease and (1) signal intensity ratio (SIR) or (2) range of movements or (3) clinical disease activity.

\section{DISCUSSION}

Radiation therapy has been used to treat thyroid eye disease since 1914, with reports suggesting that between $30 \%$ and $80 \%$ of patients treated have improvement in signs and symptoms of the disease. ${ }^{9.10}$ It appears it was most effective in patients whose duration of disease was less than 6 months and who had active ('wet' phase) soft tissue inflammatory signs. ${ }^{2}$ Periorbital swelling and other signs of ocular inflammation are known to correlate with extraocular muscle volume as determined by the use of CT. ${ }^{15,16}$ However, the superior soft tissue imaging of MRI and the relative ease of obtaining complex thin axial and coronal views compared with CT scans has led us to employ MRI as a method of assessing Graves' disease activity.

Our results show that a correlation exists between the SIR and active disease as measured according to the clinical score and restriction of extraocular movement. It would appear that extraocular muscle inflammation is more likely to be associated with a SIR of over 1.50. With the success of orbital radiotherapy for the treatment of thyroid eye disease in the early 'wet' phase, the use of the STIR sequence in MRI will be a useful adjunct in the selection of a group of patients most likely to benefit from the immunosuppressive effect of orbital radiotherapy. In particular, the SIR may be useful in conjunction with estimates of muscle cross-sectional area: a high SIR and a small muscle indicates early disease, a large muscle with a high SIR indicates late active disease and a large muscle with low SIR indicates quiescent or 'burnt-out' disease.
Having found a correlation between thyroid eye disease activity and the SIR, the next step is to assess whether this knowledge of SIR is helpful in selecting patients for lowdose orbital radiotherapy. Several patients from our study have now undergone this treatment, the results of which will be assessed and related to disease activity prior to treatment.

We would like to express our gratitude to Mr John Sparrow for his statistical expertise, Mrs Gill Bennerson for her photography and $\mathrm{Mr}$ Richard Border for providing the artwork in this manuscript and the presentation at the Oxford Congress 1993.

Key words: Extraocular muscles, MRI, STIR sequence, Thyroid eye disease.

\section{REFERENCES}

1. Char DH. Thyroid eye disease. Baltimore: Williams and Wilkins, 1985

2. Hurbli T, Devron HC, Harris J, Weaver K, Greenspan F, Sheline G. Radiation therapy for thyroid eye diseases.Am J Ophthalmol 1985;99:633-7.

3. Volpe R. Graves' disease. In: Burrow GN, Oppenheimer JH, Volpe R. Thyroid function and disease. Philadelphia: W B Saunders, 1989:214-60.

4. Gorman CA. Ophthalmopathy in Graves' disease [editorial]. N Engl J Med 1983;308:453-5.

5. Weetman AP, McGregor AM, Hall S. Ocular manifestations of Graves' disease: a review. J R Soc Med 1984;77:936-42.

6. Sergott RC, Felberg NT, Savino PJ, et al. E-rosette formation in Graves' ophthalmology. Invest Ophthalmol Vis Sci 1979;18:1245-51.

7. Trokel SL, Jakobiec FA. Correlation of CT scanning and pathological features of ophthalmic Graves disease. Ophthalmology 1981;88:553-64.

8. Kazim M, Trokel S, Moore S. Treatment of acute Graves orbitopathy. Ophthalmology 1991;98:1443-8.

9. Jones A. Orbital X-ray therapy of progressive exophthalmos. Br J Radiol 1951;24:637.

10. Ravin JG, Sisson JC, Knapp WT. Orbital radiation therapy for the ocular changes of Graves' disease. Am J Ophthalmol 1975;79:285.

11. Bartalena L, Marcocci C, Chiovato L, Laddaga M, Andreani D, Cavallacci G et al. Orbital cobalt irradiation combined with systemic corticosteroids for Graves' ophthalmopathy: a comparison with systemic steroids alone. J Clin Endocrinol Metab 1983;56:1139.

12. Brennan MW, Leone CR, Janaki L. Radiation therapy for Graves' disease. Am J Ophthalmol 1983;96:195-9.

13. Werner SC. Modification of the classification of the eye changes of Graves' disease. Am J Ophthalmol 1977; 83:725-7.

14. Mourits MPH, Koornneef L, Wiersinga WM, Prummel MF, Berghout A, Gaag RVD. Clinical criteria for the assessment of disease activity in Graves' ophthalmopathy: a novel approach. Br J Ophthalmol 1989;73:639-44.

15. Felton SE, Lee CP, Muramatsu SK, Weiner JM. Quantitative computed tomography of Graves' ophthalmopathy: extraocular muscle and orbital fat in development of optic neuropathy. Arch Ophthalmol 1985;103:213-5.

16. Hallin ES, Feldon SE. Graves' ophthalmopathy. I. Simple CT estimates of extraocular muscle volume. Br J Ophthalmol 1988;72:674-7. 